\title{
Pigeon discrimination of letters and other forms in texture displays
}

\author{
DONALD S. BLOUGH and JOHN J. FRANKLIN \\ Brown University, Providence, Rhode Island
}

\begin{abstract}
In four experiments, pigeons searched for a $3 \times 3$ matrix of small, identical forms within a $9 \times 15$ matrix composed of other repeated forms. In each session, all possible pairs of forms appeared. Percent correct and reaction time data were recorded. The stimulus sets comprised the letters of the alphabet (Experiment 1), U-shaped forms in four orientations (Experiment 2), 15 abstract forms composed of identical line segments (Experiment 3), and 3 abstract forms in four orientations (Experiment 4). Reaction times were relatively constant and uninformative. Percent correct data were analyzed by correlation, scaling, and cluster methods. The letter data correlated highly $(r=.89)$ with results from a previous study in which letters were learned as individual targets; scaling and cluster results were also very similar for the two data sets. The data for rotated Us also corresponded with previous single-target results; matrices composed of mirror-image forms were very difficult to discriminate. The data for abstract forms suggested that connectedness, orientation, and symmetry are important determiners of discrimination in this situation. Taken together, the results identify a number of features significant for pigeon form discrimination; these features include relationally defined aspects, and suggest that processes controlling discriminability here do not differ markedly from those controlling discriminability in more common learning paradigms. The method is efficient and equates exposure of test stimuli, thus minimizing the sequential effects found when stimuli are learned serially.
\end{abstract}

Like humans, birds deal expertly with objects in the visual world. Although their conceptualization of natural objects is of great interest (e.g., Herrnstein, 1979), a number of investigators have begun to analyze their perception of simple forms (e.g., D. S. Blough, 1979, 1982, 1985; P. M. Blough, 1984; Cabe, 1976; Cerella, 1977; Lea \& Ryan, 1983). These studies have typically involved the learning of specific target forms presented in relative perceptual isolation. In the present research we adopted another method: Pigeons identified a group of target forms by their difference from a background of many identical distractors. The method is efficient, it avoids order effects that are unavoidable when target forms are trained sequentially, and it may address somewhat different aspects of form perception.

A number of studies have suggested certain unique aspects of texture discrimination in humans (e.g., Caelli, 1982; Harvey \& Gervais, 1981; Julesz, 1981; Martin \& Pomerantz, 1978). For example, rapid human texture discrimination may be mediated by preattentive parallel processes that extract local features over a wide area and are relatively insensitive to the spatial relations among features (e.g., Julesz, 1981; Treisman \& Gelade, 1980). Insensitivity to spatial relations would be shown, for example, in relatively slow detection of a group of Ls embedded in an array of Ts. These letters contain the same

This research was supported in part by NSF Grant BNS-8025515. The data are drawn from the second author's Master's thesis at Brown University. Send requests for reprints to Donald Blough, Department of Psychology. Brown University. Providence, RI 02912. vertical and horizontal segments, and early texture processing might not detect the different relations between these segments, although $\mathrm{L}$ and $\mathrm{T}$ look distinctly different when attention is focused on them.

If this view is correct, texture discrimination studies may provide evidence about relatively simple primitives in the processing of forms. Explicit predictions for specific textures require the identification of primitive features or "textons" (Julesz, 1981); for example, if the differing junctions contained in $\mathrm{L}$ and $\mathrm{T}$ were themselves features, then textures of the two letters might be easily distinguished. However, even without prior determination of primitive features, one might in general expect to observe differences in the relative discriminability of two forms, depending on whether the forms were presented in relative isolation or in arrays. Experiments 1 and 2 explored this prediction by measuring the discriminability of textures composed of forms for which we already have discriminability data from pigeons. Experiments 3 and 4 examined the discriminability of forms composed of identical line segments in varying relationships.

\section{EXPERIMENF 1}

This experiment extended previous results on pigeon alphabetic letter discrimination (D. S. Blough, 1982, 1985). Its purpose was to determine the effectiveness of the texture method and to see whether the interletter similarities yielded by the texture method differ from those yielded by the specific-target method. Each stimulus was a large array composed of repeated samples of a single 
letter, within which was embedded a $3 \times 3$ target array of another letter. The pigeon's task was to find and peck the target array; errors for the various letter combinations provided a similarity matrix upon which further analyses were based.

\section{Method}

Subjects. Four male White Carneaux pigeons (Columba livia) served as subjects. The birds were experimentally naive and were about 3 years old at the beginning of training. The duration of reinforcement was adjusted to maintain the birds at $80 \%$ of free-feeding weight. Water and grit were always available in the housing cages.

Apparatus and Stimuli. Birds were trained and tested in a modified Lehigh Valley operant chamber with interior dimensions of $31 \times 35 \times 35 \mathrm{~cm}$. Directly above a centrally located feeder was a black-and-white video monitor which measured $13 \mathrm{~cm}$ diagonally. The screen was covered by a frame that contained a $6.6 \times 8.2 \mathrm{~cm}$ window, through which the stimuli were displayed. Responses were recorded from three keys made of glass microscope slides, arranged across the display window to cover each of three target areas. The chamber was illuminated by a dim overhead light and white masking noise was provided. An Atari 800 personal computer provided on-line control and data storage.

The display consisted of an array of black letters on a light gray screen, arranged in 9 rows and 17 columns. The individual alphabetic characters (Figure 1) were the same as those used by D. S. Blough $(1982,1985)$ and modeled on those of Podgorny and Garner (1979). They measured $2.0 \times 2.8 \mathrm{~mm}$ (except the letter I) and were spaced $5.5 \mathrm{~mm}$ apart horizontally and $6.0 \mathrm{~mm}$ apart vertically. Two different letters, a target and a nontarget, appeared in each display. The target letter was repeated nine times in a $3 \times 3$ array, which was located in one of three positions, each centered behind a response key. The nontarget letter filled the rest of the display. Figure 2 shows a sample array in which B is the target element and $\mathrm{Z}$ is the nontarget element. There were no visual boundaries within the display except for the faintly visible vertical edges of the response keys.

Procedure. The birds were magazine trained and shaped to peck at a black square displayed on the screen. Target letters were then introduced, and the number of background letters was increased over 8 days from none to a full matrix, while session length increased from 100 to 1,300 trials. After 13 days of further pretraining, test sessions began as described below.

A session consisted of two successive sets of 650 trials. In each set, every permutation of two different letters appeared once in random order, with the first letter serving as target and the second as background. The target array was randomly assigned to one of the three areas of the screen. An incorrect response was followed by repeated trials with the same stimulus display until a correct response occurred. Such repeat trials were preceded by a 2-sec intertrial interval.

If a correct response occurred on the first appearance of a given display, the letters turned white briefly and then disappeared, followed by houselight offset and feeder light onset for $2 \mathrm{sec}$; food was made available for, on average, 1 in 10 such trials. However, responses that had latencies of less than $0.3 \mathrm{sec}$ were not reinforced, because such rapid pecks are not well controlled by visual stimuli (e.g., D. S. Blough, 1978). Also, correct responses on repeat trials were followed by the brief color change but had no other consequences. A 3.5-sec intertrial interval followed all correct responses. Each session lasted approximately $2.5 \mathrm{~h}$; each bird was run at approximately the same time of day for 34 consecutive days.

Recorded data included reaction times for correct responses and the number of correct responses to each stimulus combination. Data for repeated trials following errors were excluded. Reaction times greater than $2.55 \mathrm{sec}$ were stored as $2.55 \mathrm{sec}$.

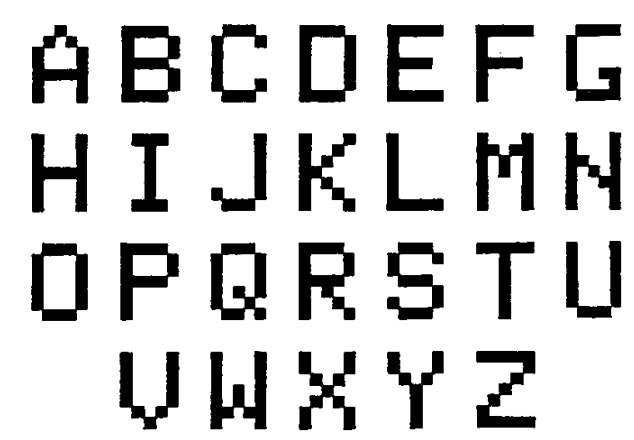

Figure 1. Format of the letters used in Experiment 1. This reproduces the format used by $D$. S. Blough $(1982,1985)$ and in a previous study of human letter similarity (Podgorny \& Garner, 1979).

\section{Results and Discussion}

Response accuracy increased rapidly over the first 7 test days, followed by relatively stable performance. Data were combined across days 8 through 34 for analyses reported here. Two sets of data, percent correct responses and median reaction times to each letter combination, were averaged across birds and sessions. As expected, these two measures were negatively correlated, but this relation was weak $(r=-.38)$. Mean median reaction time for correct responses was $908 \mathrm{msec}$, but was rather stable across stimuli $(\mathrm{SD}=41 \mathrm{msec})$. This stability, together with the large number of errors recorded, probably accounts for the low correlation between reaction time and errors. In a three-key task, one-third of correct responses may occur by chance, and as errors rise mean correct reaction times become progressively diluted with spurious values. Further analyses were based solely on percentcorrect data.

Table 1 shows percent correct responses as a function of target letter (row) and nontarget letter (column). Two scores appear for each letter pair, since each letter appeared with each other letter both as target and nontarget. The correlation between these two measures was .78. This suggests rather large differences in performance depending on whether, in individual letter pairs, a given letter was the target or the distractor, but no general pattern of such preferences was detected. To minimize preference effects in further analyses, the data matrix was folded, averaging the two percent values for each letter pair. The average interbird correlation of folded values was .75; the correlation of each bird's data with the group mean was .90. All subsequent analyses were based on the folded group means.

One view of the similarity relations between the letters, as revealed in the percent-correct data, is shown by the two-dimensional representation reproduced in Figure 3. This is a product of ALSCAL (SAS Institute, 1982), a scaling program that was adjusted to ignore all but ordinal relations in the data. The letters indicate results from the present data; arrows originate at the corresponding 


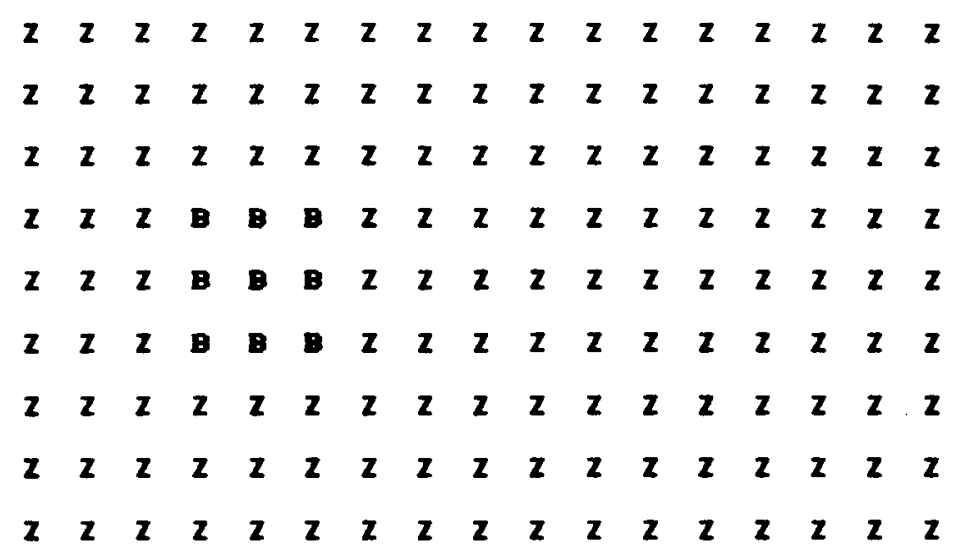

Figure 2. A display from Experiment 1 as it might appear on a single trial; 650 different displays paired each letter both as target and as background with every other letter.

positions found from the previous letter-discrimination data (D. S. Blough, 1982, 1985). The similarity of these patterns is confirmed by the high correlation $(r=.89)$ between the two similarity matrices. The present data correlated at .65 with data from human subjects judging the similarity of letters of the same format (from Podgorny \& Garner, 1979); the comparable single-letter correlation was .68 (D. S. Blough, 1985).

Like the single-letter results, the texture data shown here suggest that letters cluster by characteristic features. Such features, as well as relations to the earlier data, were clar- ified by disjoint clustering with OVERCLUS (SAS Institute, 1982), a generalization of the ADCLUS model (Shepard \& Arabie, 1979). This program found sets of clusters that simultaneously fit both the present data and the earlier single-letter data of D. S. Blough $(1982,1985)$. A solution with 25 clusters accounted for $91 \%$ of the variance in the two sets of data. The 10 clusters most heavily weighted for the present texture data are listed in Table 2, with their relative weights for the present data and for the single-letter data; possible defining features are also suggested. Nine of these clusters were among the 10 most

Table 1

Experiment 1: Mean Percent Correct

\begin{tabular}{|c|c|c|c|c|c|c|c|c|c|c|c|c|c|c|c|c|c|c|c|c|c|c|c|c|c|c|}
\hline \multirow[b]{2}{*}{ Target } & \multicolumn{26}{|c|}{ Nontarget } \\
\hline & A & B & C & D & $\mathbf{E}$ & F & $\mathbf{G}$ & $\mathbf{H}$ & I & J & $\mathbf{K}$ & L & $\mathbf{M}$ & $\mathbf{N}$ & 0 & $\mathbf{P}$ & $Q$ & $\mathbf{R}$ & $S$ & $\mathrm{~T}$ & U & V & W & $\mathrm{X}$ & $\mathrm{Y}$ & Z \\
\hline A & & 50 & 76 & 67 & 74 & 80 & 68 & 58 & 77 & 79 & 74 & 76 & 69 & 67 & 70 & 35 & 51 & 28 & 71 & 79 & 70 & 68 & 72 & 72 & 72 & 78 \\
\hline B & 45 & & 69 & 60 & 74 & & 58 & & 77 & & 9 & 74 & & 8 & & & & & & & & & & & & 72 \\
\hline $\mathrm{C}$ & 75 & 78 & & 68 & 73 & 67 & 48 & 8 & 67 & 56 & 70 & 65 & 4 & 8 & 64 & 5 & 65 & 5 & & 8 & & & & & & 70 \\
\hline D & 75 & 65 & 58 & & 80 & 80 & 67 & 6 & 70 & 71 & 80 & 75 & 82 & 82 & 31 & 66 & 46 & 78 & 76 & 79 & 5 & 7 & 80 & 80 & 75 & 82 \\
\hline E & 76 & 71 & 54 & 74 & & 33 & 47 & & 62 & 6 & 67 & 49 & 76 & 76 & 81 & 69 & 83 & 74 & 54 & 57 & 66 & 78 & 73 & 85 & 76 & 44 \\
\hline $\mathbf{F}$ & 73 & 71 & 56 & 74 & 40 & & 51 & 7 & 63 & 6 & 68 & 52 & 72 & 78 & 76 & 68 & 77 & 72 & 70 & 57 & 72 & 78 & 78 & 82 & 79 & 49 \\
\hline G & 7 & 74 & 56 & 73 & 68 & 67 & & & 77 & 6 & 68 & 66 & 77 & 79 & 9 & 67 & 74 & 72 & 60 & 76 & 76 & 81 & 81 & 78 & 76 & 66 \\
\hline H & 5 & 79 & 75 & 69 & 79 & 81 & 67 & & 71 & 7 & 64 & 77 & 59 & 45 & 7 & 71 & 67 & 63 & 5 & 79 & 65 & 62 & 70 & 61 & 56 & 82 \\
\hline I & 7 & 73 & 68 & 69 & 64 & & 63 & 74 & & 51 & 64 & 44 & & 78 & 7 & 71 & 77 & 81 & 6 & 33 & 74 & 4 & 81 & 80 & 67 & 66 \\
\hline $\mathbf{J}$ & 7 & 73 & 5 & 7 & 8 & 7 & 63 & 75 & 68 & & 65 & 56 & 77 & 76 & 7 & 66 & 72 & 76 & 70 & 68 & 60 & 7 & 76 & 79 & 63 & 70 \\
\hline $\mathbf{K}$ & 7 & 81 & 68 & 73 & 72 & 70 & 69 & 61 & 76 & 68 & & 74 & 57 & 58 & 7 & 71 & 80 & 61 & 77 & 70 & 71 & 65 & 59 & 67 & 64 & 74 \\
\hline L & 7 & 76 & 5 & 70 & 68 & 5 & 58 & 7 & 48 & 50 & 67 & & 77 & 79 & 7 & 72 & 77 & 73 & 74 & 43 & 72 & & 84 & 5 & 74 & 64 \\
\hline $\mathbf{M}$ & 7 & 77 & 78 & 74 & 76 & 7 & 76 & 5 & 78 & 76 & 55 & 79 & & 41 & 7 & 70 & 71 & 71 & & 79 & 70 & & & 73 & 65 & 82 \\
\hline $\mathbf{N}$ & 7 & 78 & 74 & 74 & 80 & 7 & 77 & 3 & 77 & 7 & 54 & 77 & 36 & & 74 & 77 & 73 & 72 & 79 & 78 & 63 & & & 54 & 9 & 74 \\
\hline 0 & 7 & 69 & 59 & 30 & 79 & 7 & 66 & 6 & 81 & 7 & 82 & 79 & 78 & 77 & & 65 & 43 & 70 & 76 & 80 & 60 & & 19 & 2 & 10 & 82 \\
\hline $\mathbf{P}$ & 4 & 45 & 65 & 69 & 71 & 7 & 59 & 6 & 80 & 7 & 75 & 70 & 76 & 82 & 72 & & 62 & 44 & 66 & 77 & 72 & & 84 & 70 & 71 & 81 \\
\hline $\mathbf{Q}$ & 5 & 61 & 70 & 46 & 81 & 8 & 6 & 7 & 82 & & 77 & 81 & 71 & 73 & 4 & 59 & & 57 & 6 & 78 & 73 & & 9 & 9 & 78 & 74 \\
\hline $\mathbf{R}$ & 45 & 40 & 72 & 69 & 76 & 8 & 62 & 6 & 80 & 7 & 73 & 80 & 73 & 80 & 6 & 38 & 66 & & 70 & 76 & 81 & & 6 & 72 & 75 & 82 \\
\hline$S$ & 6 & 64 & 59 & 72 & 68 & 6 & 48 & 7 & 74 & 7 & 79 & 78 & 73 & 74 & 69 & 62 & 73 & 58 & & 73 & 71 & 77 & 80 & 71 & 68 & 64 \\
\hline $\mathrm{T}$ & 7 & 78 & 68 & 72 & 68 & 50 & 58 & 7 & 37 & 5 & 56 & 46 & 76 & 75 & 7 & 75 & 77 & 75 & 73 & & 71 & 72 & 71 & 77 & 71 & 57 \\
\hline $\mathrm{U}$ & 7 & 79 & 77 & 62 & 80 & 79 & 65 & 65 & 77 & 61 & 74 & 71 & 65 & 70 & 5 & 78 & 67 & 72 & 82 & 76 & & 40 & 66 & 81 & 72 & 78 \\
\hline V & 7 & 79 & 70 & 54 & 78 & 83 & 72 & 5 & 81 & 7 & 69 & 73 & 66 & 64 & 55 & 75 & 71 & 76 & 75 & 79 & 30 & & 64 & 68 & 74 & 81 \\
\hline W & 74 & 76 & 72 & 75 & 81 & 80 & 73 & 47 & 72 & 73 & 55 & 77 & 33 & 41 & 78 & 76 & 74 & 72 & 79 & 74 & 67 & 61 & & 66 & 68 & 79 \\
\hline $\mathrm{X}$ & 56 & 70 & 70 & 68 & 78 & 81 & 68 & 42 & 68 & 6 & 57 & 74 & 6 & 57 & 71 & 65 & 64 & 56 & 73 & 78 & 73 & 62 & 53 & & 32 & 77 \\
\hline $\mathrm{Y}$ & 65 & 67 & 71 & 74 & 81 & 71 & 65 & 4 & 6 & 5 & 5 & & 6 & 5 & 69 & 6 & 6 & 65 & 71 & 69 & 63 & 59 & 59 & 39 & & 77 \\
\hline Z & 84 & 74 & 72 & 79 & 56 & 47 & 50 & 81 & 68 & 67 & 72 & 60 & 81 & 82 & 78 & 77 & 79 & 73 & 71 & 69 & 75 & 74 & 74 & 80 & 79 & \\
\hline
\end{tabular}




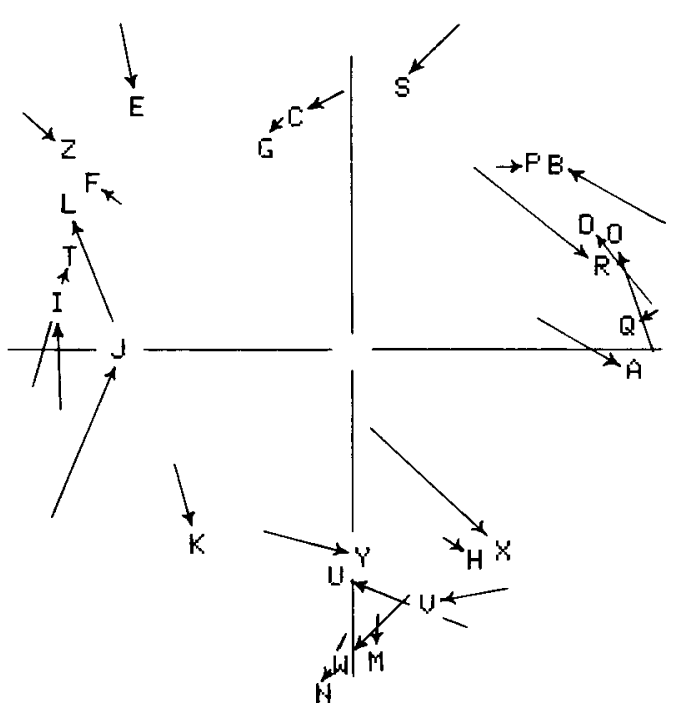

Figure 3. ALSCAL two-dimensional representation of interletter distances based on pigeon error data. Letters by arrow heads show positions based on the present study; tails of the arrows show positions based on data from a previous study (D. S. Blough, 1982, 1985).

heavily weighted in both data sets; the exception is Cluster 8 , which ranked 12 th for the single-letter data.

Of the 25 clusters computed, 3 were much more heavily weighted for the single-letter data than for the texture data. These were the following, together with their weights: CG $(16.0,3.1)$, FIKTY $(8.5,0.5)$, and $\operatorname{RSXZ}(5.4,0)$. There were no differences this large in the opposite direction.

We suggested above that the present texture-discrimination data might reflect primarily those immediate perceptual properties that permit one group of letters to stand out from another. In contrast, the single-letter discrimination procedure involved a response to a specific remembered target form in a task that might seem to demand more attention. The correspondence of the data from these two methods, shown by the .89 correlation between them (see also Table 2) suggests that the variance in both tasks flows largely from common sources, which are not strongly influenced by the texture format. That is, "texture segregation" did not yield clearly different results than did a comparison of widely separated single forms. We return to this matter in the General Discussion section.

\section{EXPERIMENT 2}

Some experiments with humans have used simple $U$ forms to evaluate the effect of texture element orientation (e.g., Julesz, Gilbert, Shepp, \& Frisch, 1973). The effect of orientation on the recognition of such forms presented singly and in a three-choice task has also been measured in pigeons (Todrin \& D. S. Blough, 1983). Experiment 2 evaluated pigeon discrimination of these forms in texture arrays. As with the letter stimuli, we were interested in discovering whether the texture method yields the same pattern of confusions as the classic discrimination method, and whether the confusions are like those seen in human perception of textures composed of these forms.

\section{Method}

Subjects. The 4 pigeons used in Experiment 1 served as subjects. They were maintained as before.

Apparatus and Stimuli. The apparatus was the same as that used in Experiment 1. The stimuli were U-shaped, open-ended $2.4 \times 2.4 \mathrm{~mm}$ squares, presented upright and in $90^{\circ}, 180^{\circ}$, and $270^{\circ}$ rotations. The upright and inverted Us will be referred to as "updown mirror images," the other pair as "left-right mirror images." These $\mathrm{U}$ forms were arrayed in texture targets and backgrounds as in Experiment 1, with the individual forms spaced $5.1 \mathrm{~mm}$ apart horizontally and $6.4 \mathrm{~mm}$ apart vertically.

Procedure. Because several months separated Experiments 1 and 2 , the birds were first retrained with two sessions on the testing procedure used in Experiment 1. Experiment 2 then began. The procedure was the same as that for Experiment 1, with the following exceptions: a session consisted of 100 blocks of 12 trials each; in each block, every permutation of two different $U$ characters appeared once in random order; the birds were run for 5 consecutive days.

\section{Results and Discussion}

Data were combined across sessions and birds. Accuracy increased dramatically between the first and second test sessions and then was relatively stable. Mean reaction time for correct responses was almost the same as that in Experiment $1(908 \mathrm{msec})$. Although the standard deviation across stimuli was small $(26 \mathrm{msec})$, reaction times were significantly related to form: The birds responded more rapidly to nonmirror-image pairs than to mirror-image pairs $(p<.01)$, and more rapidly to updown pairs than to left-right pairs $(p<.01)$. Corresponding differences appeared in percent-correct data, which are summarized in Table 3 . These data, averaged across target/nontarget conditions and across birds, yielded $76.8 \%$ correct for nonmirror pairs, $47.5 \%$ for up-down mirror pairs, and $44.5 \%$ for left-right pairs (chance $=$ $33.3 \%)$. Each of these values differed significantly from the others $(p<.01)$.

These results replicate in pigeons the finding in humans that two textures composed of $U$ forms are much more difficult to isolate when the texture elements are mirror

Table 2

Experiment 1: Ten Most Heavily Weighted Clusters of Letters

\begin{tabular}{|c|c|c|c|}
\hline \multirow[b]{2}{*}{ Cluster } & \multicolumn{2}{|c|}{ Cluster Weights } & \multirow{2}{*}{$\begin{array}{c}\text { Possible Feature } \\
\text { Names }\end{array}$} \\
\hline & Texture & Single-Letter & \\
\hline $1 \mathrm{UV}$ & 28.2 & 30.0 & open up, open center \\
\hline 2 A B P R & 23.0 & 23.8 & small loop \\
\hline 3 D O Q & 22.5 & 20.4 & closed, open center \\
\hline $4 \times Y$ & 20.7 & 15.0 & small open up \\
\hline $5 \mathrm{M} \mathrm{N} \mathrm{W}$ & 20.4 & 12.5 & straight, open up \\
\hline $6 \mathrm{EF}$ & 18.5 & 21.5 & straight, open right \\
\hline $7 \mathrm{HKMNWXY}$ & 13.3 & 15.9 & open up, cross cente \\
\hline $8 \mathrm{EFG} \mathrm{SZ}$ & 12.7 & 6.9 & open right \\
\hline 9 F I L T & 12.6 & 12.3 & single vertical \\
\hline $10 \mathrm{BQ}$ & 11.5 & 11.4 & closed, inner line \\
\hline
\end{tabular}


Table 3

Experiment 2: Mean Percent Correct

\begin{tabular}{ccccc}
\hline & \multicolumn{4}{c}{ Nontarget } \\
\cline { 2 - 5 } Target & {[} & $\square$ & $\sqsupset$ & $\sqcup$ \\
\hline$\square$ & & 77 & 44 & 79 \\
$\sqcap$ & 78 & & 74 & 46 \\
$\sqsupset$ & 45 & 74 & & 76 \\
$\sqcup$ & 79 & 49 & 77 & \\
\hline
\end{tabular}

images than when they are not (Julesz et al., 1973). Various models of form recognition predict such confusion, notably the texton theory Julesz (1981) mentioned above. The mirror-image pairs in the present study have the same number and size of horizontal and vertical bars, and have the same number of line endings; they differ only in the spatial relations of these elements. Thus, texturediscrimination theories that stress insensitivity to spatial relations predict very large confusion differences between mirror pairs and nonmirror pairs. Conversely, the data embarrass models based on superposition or template matching, since here all pairs of Us overlap by the same amount (two-thirds).

The findings from the present texture task also agree with those based on pigeon discrimination of individually learned U forms (Todrin \& D. S. Blough, 1983). As in Experiment 1, this suggests a common source of errors in the two tasks. The correspondence extends both to the large mirror/nonmirror difference and to the small superiority of up-down to left-right mirror-image discrimination. The latter finding coincides with much evidence from humans and other species, and the special nature of left-right confusion has generated a literature of its own (see Bornstein, 1982, and Corballis \& Beale, 1976, for reviews). Such observations vex simple models of form recognition; several aspects of this matter were discussed by Todrin and D. S. Blough (1983).

\section{EXPERIMENT 3}

The forms used in Experiments 1 and 2 were chosen primarily because they had been used in previous experiments whose results could be compared with those from the texture method. The forms used in Experiment 3 are new; they were designed to limit the possible controlling aspects. The forms appear in Figure 4; all share three identical long line segments in one orientation and, at right angles to these, two short segments. The forms differ only in the relative location of the short segments. This set of forms was inspired largely by the texton theory of Julesz (Julesz \& Bergen, 1983) and the feature-integration theory of Treisman \& Gelade (1980). As mentioned above, these theories share the idea that simple features are registered quickly and in parallel across the visual field, whereas the detection of relationships among features, typically required for specific object identification, is a longer process requiring focal attention. Both theories have been tested in texture and search tasks somewhat like that used here. Among their elementary features, or tex- tons, Julesz and Bergen listed "blobs" of a given size and orientation, terminators (e.g., line endings), and crossings. Textures differing sufficiently in the type or density of these and possibly other elements should be quickly and easily discriminated. The two theories also suggest that the rapid nonfocal texture-discrimination process is insensitive to the relative position of such local features within the individual forms that compose a texture. Thus, it should be hard to discriminate two textures having the same density of line segments with given size and orientation and with the same terminators and crossings, regardless of the relations among these elements. Textures composed of forms that differ in one or more of these respects should be easier to discriminate.

\section{Method}

Subjects. The 4 pigeons used in Experiment 1 served as subjects. They were maintained as before.

Apparatus. The apparatus was the same as that used in Experiment 1 .

Stimuli. The stimulus forms appear in Figure 4. The forms were all composed of the same three long and two short line segments; in Condition 1 these were, respectively, horizontal and vertical. Only the two verticals varied in location; in some forms they fused to a single longer vertical. The forms differed from two to six in number of terminators (line endings) and in junctions, including corners and $\mathrm{T}$-junctions. Certain groups of forms had identical local features; that is, they had the same number and orientation of lines, junctions, corners and line endings. These groups comprised forms numbered $1,3,11$, and $13 ; 2,4,12$, and 14 ; and 5, 6, 9, and 10 in Figure 4. If left-right orientation is disregarded, Forms 7 and 8 have identical local features and other groups also coalesce. Form 15 had more terminators than the others and different junctions. For human observers, a major global difference in the forms has to do with whether all parts of the form are hooked together; we term this "connectedness," and it distinguishes Forms 1-6 from Forms 7-15. Note, for example, that a match can be found for each local area in Forms 5 and 9, yet they differ in connectedness. The implications of these differences are discussed below.

The forms used in Condition 1 appeared in the orientation shown in Figure 4; in Condition 2, they appeared in $90^{\circ}$ rotation. In both conditions, the forms appeared in an array as black on a light gray ground. They were $2.8 \mathrm{~mm}$ square and were spaced $4.7 \mathrm{~mm}$ apart

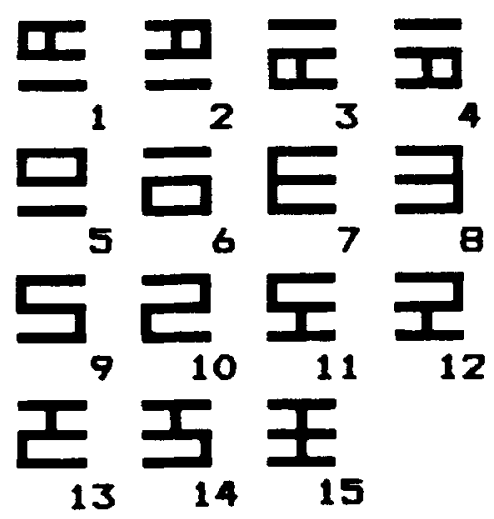

Figure 4. The set of forms used in Experiment 3. The forms are all composed of the same five line segments. The orientation used in Condition 1 is shown; $90^{\circ}$ rotations were used in Condition 2. Forms 5, 7, and 9 were used in Experiment 4. 
horizontally and $6.0 \mathrm{~mm}$ apart vertically, in 9 rows and 17 columns. As in earlier experiments a repeated target form filled a $3 \times 3$ array directly behind one of the three glass keys; a nontarget form filled all other positions.

Procedure. The birds were run first on Condition 1 and then on Condition 2. The procedure was the same as that for Experiment 1 except in the following respects: Condition 1-Each bird first received two warm-up sessions with the alphabetic task of Experiment 1 . Subsequently, the arrays were composed from the forms shown in Figure 4. Each session consisted of four blocks of 210 trials each. In each block, every pair of forms appeared twice, once with each form as target and the other as background; order was randomized. Daily sessions lasted about $105 \mathrm{~min}$. Percent correct data were recorded, and birds were run for 10 sessions after reaching a criterion of $50 \%$ correct responses for all trials in a session. Condition 2-The forms in Figure 4 were rotated $90^{\circ}$. Otherwise, the procedure was the same as that for Condition 1, except that warmup sessions were omitted.

\section{Results and Discussion}

Percent correct data from the 10 sessions following criterion for each condition were analyzed; because error rates were high, reaction times were not analyzed. Tables $\mathbf{4}$ and $\mathbf{5}$ show mean-percent data for each condition. The overall percent correct scores were low, with a mean of $59 \%$ for Condition 1 and $55 \%$ for Condition 2 . Although some forms yielded better performance than others, even the best performance was only $82 \%$ in Condition 1 and $75 \%$ in Condition 2 . This finding suggests that the forms may have differed insufficiently for the target texture to "pop out" obviously from the background.

The correlation between target scores and their corresponding nontarget scores was .69 for Condition 1 and .73 for Condition 2 . These low values reflect some systematic asymmetries in the data. Most striking of these was the ease with which discriminations were made when the $S$ forms ( 9 and 10) were targets, relative to discrimination when these forms were distractors: This difference appeared in 51 and 52 individual pairings involving these two forms across the two conditions, with a mean difference in correctness of $13 \%$. At the opposite extreme were Forms 1-4 and 11-15; for all of these, performance was

Table 4

Experiment 3, Condition 1: Mean Percent Correct

\begin{tabular}{cccccccccccccccc}
\hline & \multicolumn{110}{c}{ Target } & 1 & 2 & 3 & 4 & 5 & 6 & 7 & 8 & 9 & 10 & 11 & 12 & 13 & 14 & 15 \\
\hline 1 & & 34 & 32 & 32 & 56 & 57 & 69 & 66 & 57 & 54 & 62 & 69 & 64 & 74 & 69 \\
2 & 34 & & 33 & 37 & 65 & 56 & 63 & 66 & 66 & 55 & 60 & 66 & 63 & 60 & 73 \\
3 & 39 & 36 & & 38 & 68 & 54 & 72 & 68 & 58 & 59 & 64 & 64 & 62 & 61 & 74 \\
4 & 40 & 37 & 34 & & 61 & 54 & 70 & 64 & 62 & 61 & 67 & 65 & 74 & 60 & 68 \\
5 & 71 & 72 & 74 & 77 & & 36 & 74 & 72 & 54 & 58 & 81 & 68 & 75 & 73 & 80 \\
6 & 76 & 67 & 75 & 75 & 39 & & 75 & 75 & 55 & 58 & 76 & 83 & 76 & 70 & 84 \\
7 & 75 & 81 & 78 & 79 & 76 & 75 & & 42 & 39 & 41 & 38 & 49 & 39 & 49 & 54 \\
8 & 81 & 76 & 76 & 73 & 72 & 81 & 47 & & 35 & 41 & 56 & 39 & 48 & 35 & 58 \\
9 & 77 & 75 & 82 & 75 & 71 & 64 & 51 & 47 & & 32 & 55 & 52 & 57 & 58 & 77 \\
10 & 80 & 78 & 81 & 81 & 75 & 71 & 55 & 51 & 33 & & 63 & 54 & 60 & 54 & 74 \\
11 & 81 & 74 & 72 & 74 & 79 & 73 & 36 & 50 & 38 & 42 & & 37 & 32 & 45 & 48 \\
12 & 80 & 74 & 80 & 73 & 73 & 72 & 49 & 45 & 40 & 40 & 48 & & 54 & 33 & 50 \\
13 & 76 & 70 & 68 & 72 & 77 & 74 & 38 & 46 & 40 & 41 & 31 & 47 & & 46 & 50 \\
14 & 73 & 75 & 74 & 76 & 73 & 75 & 52 & 39 & 49 & 41 & 53 & 36 & 42 & & 56 \\
15 & 72 & 70 & 64 & 68 & 62 & 71 & 47 & 45 & 40 & 38 & 38 & 39 & 36 & 32 &
\end{tabular}

Table 5

Experiment 3, Condition 2: Mean Percent Correct

\begin{tabular}{|c|c|c|c|c|c|c|c|c|c|c|c|c|c|c|c|}
\hline \multirow[b]{2}{*}{ Target } & \multicolumn{15}{|c|}{ Nontarget } \\
\hline & 1 & 2 & 3 & 4 & 5 & 6 & 7 & 8 & 9 & 10 & 11 & 12 & 13 & 14 & 1 \\
\hline 1 & & 47 & 30 & 43 & 61 & 61 & 62 & 66 & 63 & 67 & 45 & 57 & 55 & 67 & \\
\hline 2 & 41 & & 39 & 40 & 57 & & 65 & 7 & 54 & 4 & & & 99 & 49 & \\
\hline 3 & 37 & 49 & & 50 & 56 & 62 & 60 & 70 & 58 & 57 & 2 & 56 & 53 & 57 & \\
\hline 4 & 4 & 32 & 39 & & 64 & 52 & 65 & 59 & 56 & 59 & & 40 & 53 & 2 & \\
\hline 5 & 6 & 64 & 61 & 66 & & 37 & 50 & 7 & 34 & 32 & & 7 & 2 & 5 & \\
\hline 6 & 6 & 73 & 61 & 64 & 34 & & 47 & 8 & 41 & 29 & & 2 & 59 & & \\
\hline 7 & 69 & 71 & 69 & 75 & 58 & 51 & & 53 & 34 & 49 & 42 & 1 & 42 & 0 & \\
\hline 8 & 71 & 62 & 75 & 70 & 53 & 56 & 39 & & 42 & 41 & 57 & 41 & 62 & 47 & \\
\hline 9 & 6 & 7 & 72 & 73 & 44 & 42 & 48 & 55 & & 37 & 58 & 4 & 50 & 54 & \\
\hline 10 & 73 & 78 & 67 & 76 & 41 & 41 & 50 & 49 & 38 & & 58 & 7 & 59 & 58 & \\
\hline & 56 & 68 & 58 & 65 & 67 & 62 & 48 & 63 & 52 & 57 & & 44 & 36 & 43 & \\
\hline 1 & 60 & 56 & 63 & 56 & 65 & 61 & 60 & 47 & 48 & 48 & 38 & & 44 & 33 & \\
\hline & & 7 & 50 & 70 & 69 & 63 & 45 & 63 & 54 & 50 & 37 & 5 & & 51 & \\
\hline & & 5 & 6 & 6 & 61 & & 55 & 4 & 51 & 55 & 44 & & 44 & & \\
\hline 15 & 55 & 60 & 60 & 3 & 71 & 00 & 61 & ב & 60 & 56 & 35 & 43 & 41 & 41 & \\
\hline
\end{tabular}

better if the form was a distractor than if it was a target. This difference was recorded in 90 of 108 pairings between forms in this group and forms not in this group, with a mean difference of $8 \%$.

A human observer might relate these target-distractor asymmetries to apparent complexity: The $S$ is the only form that can be seen as a single, unbroken line, whereas forms in the other group all have short interior segments that create a complex appearance. Some experiments with human subjects have also generated target-distractor asymmetries. For example, detection of an unfamiliar target on a familiar background is faster than the reverse (e.g., Martin \& Pomerantz, 1978; Reicher, Snyder, \& Richards, 1976); also, when a target is defined by the presence of a feature that is not in the background, discrimination is easier than under the reverse condition (e.g., Beck, 1973). Neither of these effects is reflected in the present data, and because the symmetry of similarity relationships is of considerable theoretical importance (e.g., Tversky, 1977), it would be worthwhile to attempt a more precise specification of target-distractor asymmetry by the use of other form sets.

Although the differences just considered were common to Conditions 1 and 2, the data from the two conditions (matrices in Tables 3 and 4) correlated only .71. This rather low value is largely attributable to a single factor: forms that showed partial or total symmetry around the vertical axis were discriminated considerably better, relative to the mean discrimination in a given condition, than their rotated variations, which were symmetrical around a horizontal axis. Thus, Forms 5 and 6 were vertically symmetrical in Condition 1 and became horizontally symmetrical in Condition 2, whereas the reverse was true for Forms 7 and 8 . In 40 instances, these forms were paired with forms that were asymmetrical in both Condition 1 and Condition 2. The pair was better discriminated, relative to the mean performance in each condition, in 25 instances when it included a vertically symmetrical form; the horizontally symmetrical version was better discriminated in only 4 instances. (Eleven cases were "tied," with 
less than 3\% difference between conditions.) Interestingly, forms $11,12,13$, and 14 , which are locally symmetrical about an interior short segment, were also better discriminated in Condition 1. These results may be related to others that identify vertical symmetry as an important factor in human form perception (e.g., Fox, 1975).

The rotation of forms from Condition 1 to Condition 2 converted a number of left-right mirror-image discriminations to up-down discriminations (Forms 1 and 2, 3 and 4,7 and 8,11 and 12,13 and 14). In agreement with the results of Experiment 2, each of these pairs was better discriminated in Condition 2 (up-down), relative to the mean performance in their respective conditions.

For further analysis, the corresponding target and nontarget percentages were averaged for each set of data. These folded data from Conditions 1 and 2 were input to the ALSCAL multidimensional scaling program (SAS Institute, 1982), which produced the output plotted in Figure 5. This analysis used the INDSCAL option to fit data from Condition 1 and Condition 2 simultaneously.

The dimensional organization here is fairly clear: Disconnected forms fall to the left and connected forms to the right in Figure 5, whereas forms without an interior segment are toward the top and those with an interior segment are toward the bottom. The horizontal dimension was more important than the vertical in accounting for the data of Condition 1 (weights .88 vs. .38), whereas the opposite was true for Condition 2 (weights .55 vs. .72).

Figure 5 suggests that categorization, rather than dimensional representation, may best describe the data. This matter was explored by cluster analysis using OVER-

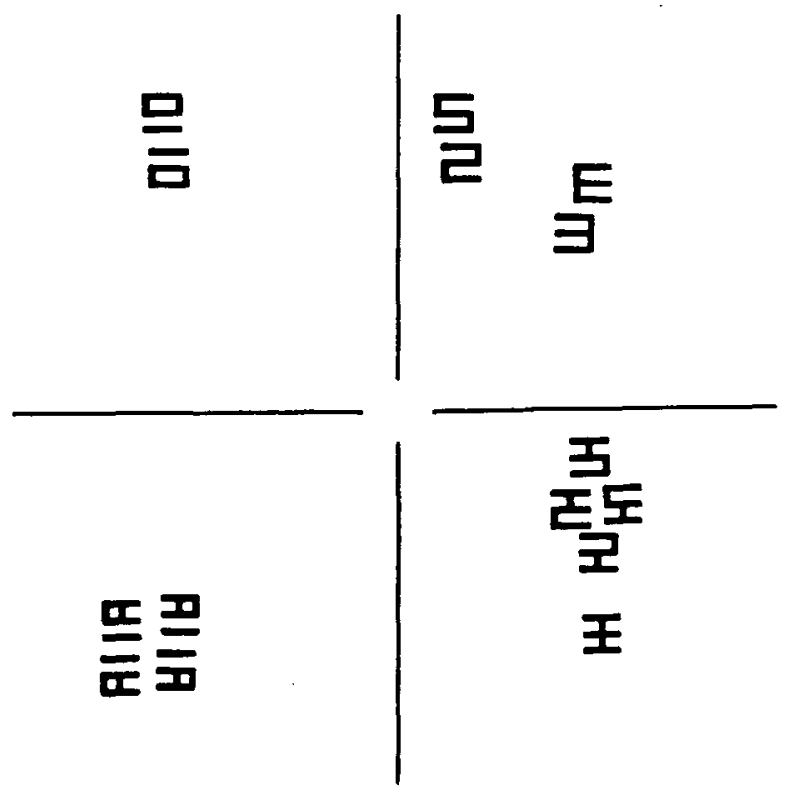

Figure 5. INDSCAL representation of interform distances based on mean error data from Experiment 3. The "interval" option was used in this scaling to separate crowded forms. Note that the forms are shown as they appeared in Condition 1 , but the scaling was based on both conditions.
CLUS (SAS Institute, 1982), a procedure that, like INDSCAL, was used to simultaneously fit the data from the two conditions and provide weights for each. The results for eight clusters are shown in Figure 6, which depicts the forms in each cluster together with their weights for each condition and a possible description of the cluster. (These eight clusters accounted for $88 \%$ of the data variance; the incremental improvement for solutions with more clusters was less than $1 \%$ per added cluster.)

The cluster analysis supports the categorization suggested by Figure 5 and adds additional detail. As suggested by the two-dimensional picture, connectedness is heavily weighted and accounts for much of the variance; forms in Cluster 1 are connected, those in Clusters 2 and 7 disconnected. The interior segment accounts for the vertical dimension in Figure 5; it is common to forms in Clusters 2 and 8, and is absent in Clusters 3, 6, and 7. Cluster 4 is characterized by a short segment at the right and Cluster 5 by one at the left; alternatively, either of these may be seen as a $C$ configuration facing left or right. It is interesting that quite discriminable forms appear to cluster on the basis of these mirror-image features, given the tendency demonstrated in Experiment 2 (and in Todrin \& D. S. Blough, 1983) for pigeons to confuse mirrorimage forms. Finally, grouping defined by identity of local features (including segments, corners, junctions, and line endings) but not by relative position did not play a prominent role in the clustering, characterizing only Group 3 and its subset Group 7.

These results confirm the importance of relationally defined features that seemed evident in Experiment 1, in which different local features such as straight lines or loops appeared to work with oriented configurations such as "open up" to account for much of the data. Here, with the number of local features severely limited, and orientation of line segments equated, the pigeons' discriminations appear to be affected by several different relational aspects, including connectedness, relative location of short segments (left, interior, right), and symmetry about the vertical axis. These relational possibilities strongly constrain any attempt to model such results. In particular, they appear very difficult for a superposition or template type of model to handle. It is further evident that, although the forms differ in some respects in terms of previously defined textons (e.g., in number of visible line endings), it is difficult to account for the data on the basis of such simple nonrelational features.

\section{EXPERIMENT 4}

Displays such as those used in Experiments 1-3 do not control for some possible configurational factors that might influence texture discrimination. For example, the edge of a target texture might be enhanced by a row of colinear line segments that stand out against a series of broken segments in an adjacent background row. Some experimenters have displayed forms in random positions and orientations in order to reduce the possibility of such 
Cluster

\section{Meights Description \\ $\operatorname{con} 1 \operatorname{con} 2$}

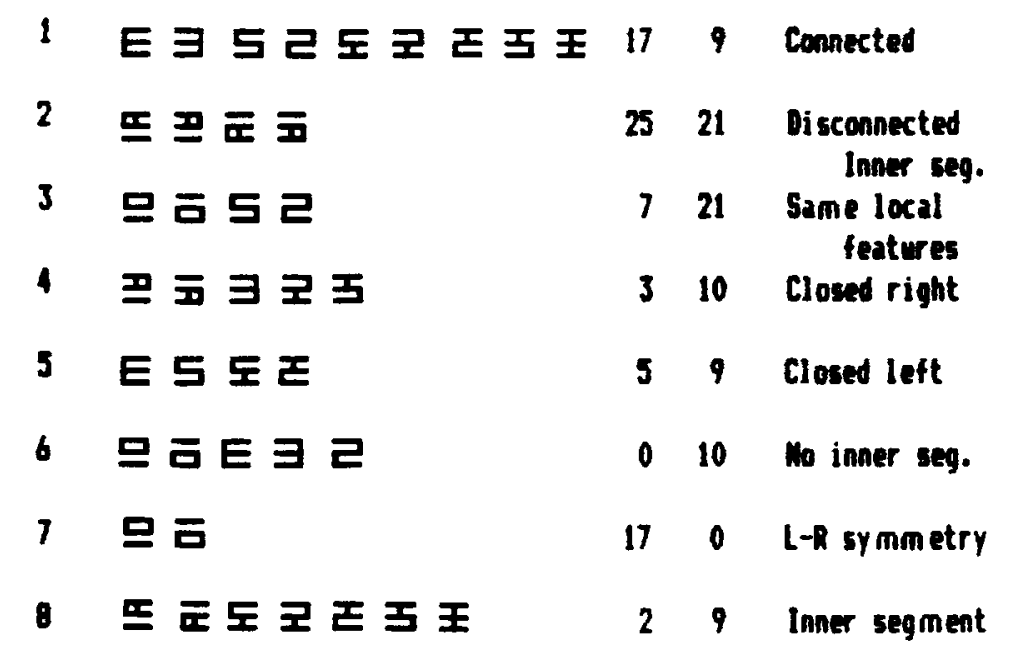

Figure 6. Clusters derived by OVERCLUS from the error data of Experiment 3, together with weights for the two experimental conditions. Most of the cluster descriptions have plausible alternatives.

effects. Varying orientation within the display also removes orientation itself as a defining variable, as it clearly was in Experiment 3. For example, Forms 2, 3, and 4 were rotations and/or reflections of Form 1, and form classification depended in part on orientation (Figure 6).

Of particular concern was the relatively great importance of connectedness in determining the results of Experiment 3 . Because rapid texture discrimination is supposed to be insensitive to such variables (e.g., Julesz \& Bergen, 1983; Triesman \& Gelade, 1980) we wished to confirm that our findings were not somehow related to the orientational indentity of all forms displayed in Experiment 3. We chose Forms 5, 7, and 9 from the set used in Experiment 3 (Figure 4). Forms 7 and 9 were connected, Forms 5 and 9 shared the same local features, and Forms 5 and 7 differed in both respects. These forms appeared in a random mixture of four orientations, a presentation that should reduce the influence of configural factors along texture boundaries and that precludes the use of orientation itself as a cue. The key question was whether these adjustments would change the relative importance of connectedness and local features in determining the results.

\section{Method}

Subjects and Apparatus. The 4 pigeons used in Experiment 1 served as subjects. They were maintained as before. The apparatus was the same as that used in Experiment 1.

Stimuli. Forms labeled 5, 7, and 9 in Figure 4 served as stimuli. Each of these forms could appear in one of four rotations: $0^{\circ}, 90^{\circ}$, $180^{\circ}$, or $270^{\circ}$ from the orientation shown in Figure 4. Their characteristics were otherwise as in Experiment 3.

Procedure. Two warm-up sessions were run, as in Experiment 3, Condition 1. In subsequent sessions, two of the three forms appeared on each trial, instances of one composing the target texture and the other the nontarget texture. Within these textures, each form appeared randomly in one of its four possible orientations. A session consisted of 100 blocks of six trials; each permutation of the six forms appeared once in each block.

\section{Results and Discussion}

Percent correct data were analyzed for each bird from the five sessions following a criterion of $50 \%$ correct response overall. The outcome was quite similar to that for the same pairs of forms in Experiment 3; specifically, the mean folded data from the two conditions of Experiment 3 compared with the mean folded data from Experiment 4 as follows (Experiment 3 data first): For Forms 5 and $7,65 \%$ and $62 \%$; for Forms 5 and $9,51 \%$ and $54 \%$; for Forms 7 and $9,46 \%$ and $40 \%$.

It appears that the pattern of results found in Experiment 3 for these forms did not strongly depend on either specific orientation or interform configurational effects. The data thus confirm that both connectedness and local features affect performance of this task: the forms that differed in both ways ( 5 and 7) were best discriminated in both experiments, those differing only in connectedness ( 5 and 9) were next best discriminated, and those differing only in local features ( 7 and 9 ) were most poorly discriminated.

If anything, the relative importance of connectedness increased in Experiment 4, as the pairs that differed in this respect alone increased in relative discriminability. This particular pair of forms ( 5 and 9) have been shown in human subjects to resist texture segregation (Julesz \& Bergen, 1983), a finding used to support the contention that texture processing is insensitive to such relational 
aspects. However, our finding that this variable is relatively important in the present situation by no means demonstrates that pigeons differ from humans in their processing of textures. Indeed, the generally poor discrimination of the forms suggests the contrary: these displays may have forced the subjects to use slow, attentive search, because feature differences among the forms were not prominent enough to induce rapid texture segregation. In Experiments 3 and 4, all the forms had three long line segments at right angles to two short segments; feature differences were confined to the number of line endings and the sorts and numbers of junctions. Clearly, further data are needed to define the role of features in pigeon texture segregation.

\section{GENERAL DISCUSSION}

The principal results of these experiments may be summarized as follows. First, the relative similarity for the pigeon of a number of forms was described; the data variance was rather well accounted for by clusters associated with relatively simple characteristics of the forms, some of which, at least, required relational definition. Second, the texture discrimination method proved an efficient way to evaluate the relative similarity of many pairs of forms, without requiring training on specific pairs. Third, the results suggest that the processes determining similarity among forms such as these are similar, whether measured by the texture method or by a more traditional discrimination procedure.

Each of these findings deserves further comment. The present results confirm and extend previous data (D. S. Blough, 1985) that permit discrimination errors to be ascribed largely to joint control by a number of simple aspects or features (see Table 2; Figure 6). This does not mean that form recognition actually involves parsing into such features, and it remains questionable whether feature analysis is the best way to model form processing in pigeons (see D. S. Blough, 1985). However, the data constrain any theory; for example, the processes here clearly are not blind to orientation or connectedness, and Experiment 3 suggested that symmetry about the vertical axis may be significant as well (cf. Delius \& Nowak, 1982).

The success of the texture discrimination method is worth noting, because most previous studies of animal form perception have involved laborious training on a succession of specific form discriminations. More abstract tasks, such as indicating sameness or difference across many form pairs, are difficult if not impossible for pigeons (see D'Amato, Salmon, \& Colombo, 1985; but see also Lombardi, Fachinelli, \& Delius, 1984). We have repeatedly failed to produce oddity learning in pigeons on a three-choice task very much like that used here, except that a single form rather than an array appeared behind each key (unpublished). Because the abstraction of oddity seems so difficult for these birds when only three stimuli are present, it is tempting to conclude that the tex- ture task involves a different level of processing, presumably presenting "difference" at a more immediate or perceptual level. The pigeon's limited ability to abstract (cf. Premack, 1978) may be less taxed here, because an odd patch of texture stands out from its background more directly than does the difference between an odd form and two identical forms nearby.

Despite this suggestion that the texture display provides more effective perceptual input than that from isolated forms, the similarities measured here seem comparable to those measured through specific target training. Thus, if texture segregation is playing a unique role, we have been unable to detect its influence in the similarity matrices. Results from Experiments 1 and 2 correlated highly with those from choice tasks in which only a single target form and two distractors appeared simultaneously (D. S. Blough, 1982, 1985; Todrin \& D. S. Blough, 1983). Furthermore, spatial relations among form segments clearly affected the data of Experiments 3 and 4, although such relations are thought to play a minimal role in human texture segregation (e.g., Julesz, 1981; Treisman \& Gelade, 1980). Taken together, these results suggest that the main process behind the pigeons' performance in the present experiments may be more like attentive inspection in humans than it is like the automatic, preattentive texture segregation identified in some texture and search tasks used previously with human subjects.

Such a disposition toward attentive inspection may not be surprising from a human perspective, for many of the displays used here are not very conducive to rapid texture segregation. The textures were rather coarse, each element subtending about $2^{\circ}$ of visual angle at the bird's eye, and many form pairs may not have differed enough to generate texture segregation in the sense reported by human subjects. In consequence, a human observer might use a mixed perceptual strategy in such a situation; that is, when textures are sufficiently different, they would segregate automatically and a rapid response would follow, whereas similar forms would provoke timeconsuming item-by-item search. This strategy has much in common with the two-stage model of visual search proposed by Hoffman (1978).

Such a mixed strategy predicts large variations in reaction time, long times for similar forms, short times for dissimilar ones. This makes it an unlikely account of our data, for our reaction times were quite constant, with a standard deviation of only $41 \mathrm{msec}$ around the mean of $908 \mathrm{msec}$ across the data set of Experiment 1 . Thus the birds may have allocated an essentially fixed time to their search for a target and then responded, consequently making many errors if the target was hard to find. This is the "fast-guess" strategy that is seen in some human psychophysical tasks and that often characterizes pigeon discriminative behavior (e.g., D. S. Blough, 1978). It is worth noting, however, that the tradeoff of speed and accuracy is a function of relative payoff; with three response keys, our birds could be correct by chance one-third of the time, and they probably took this chance rather than 
prolong a difficult search. Procedures with more response alternatives reduce the reward for taking a chance, and can produce high accuracy and informative reaction times (e.g., D. S. Blough, 1979, in press; P. M. Blough, 1984).

A fast-guess account does leave some room for variation on the mixed strategy mentioned above. Possibly, for some displays, texture segregation in the sense reported by humans was immediate and guided rapid, accurate responding. On many displays, we would assume, such segregation failed; however, instead of slowly searching the display, the pigeons looked only for a moment and then responded correctly if the target form had been identified in that brief period. Assuming, as we have argued, that the displays of Experiment 4 are inadequate to generate texture segregation, the present data are consistent with this mixed account, although they are also consistent with the idea that no texture segregation occurred in any experiment.

It remains something of a paradox that the texture task worked so well for pigeons, yet we find no evidence that perceptual texture segregation was what made the task easy. Zentall, Hogan, Edwards, and Hearst (1980) found that oddity learning in pigeons was enhanced by a manyitem display, and they suggested the enhancement of figure-ground relations as a possible influence. Something of this sort may be involved in the present instance, although further information is required in order to define its nature more specifically.

\section{REFERENCES}

Beck, J. J. (1973). Similarity grouping of curves. Perceptual \& Motor Skills, 36, 1331-1341.

BLOUGH, D. S. (1978). Reaction times of pigeons on a wavelength discrimination task. Journal of the Experimental Analysis of Behavior, 30, 163-167

BLough, D. S. (1979). Effects of the number and form of stimuli on visual search in the pigeon. Journal of Experimental Psychology: Animal Behavior Processes, 5, 211-223.

Blough, D. S. (1982). Pigeon perception of letters of the alphabet. Science, 218, 397-398.

Blough, D. S. (1985). Discrimination of letters and random dot patterns by pigeons and humans. Journal of Experimental Psychology: Animal Behavior Processes, 11, 261-280.

Blough, D. S. (in press). Form similarity and categorization in pigeon visual search. In M. L. Commons, R. J. Herrnstein, \& S. Kosslyn (Eds.), Quantitative analyses of behavior: Pattern recognition and concepts in animals, people, and machines. Hillsdale, NJ: Erlbaum.

Blough, P. M. (1984). Visual search in pigeons: Effects of memory set size and display variables. Perception \& Psychophysics, 35, 344-352.

Bornstein, M. H. (1982). Perceptual anisotropies in infancy: Ontogenetic origins and implications of inequalities in spatial vision. In H. W. Reese, L. P. Lipsitt (Eds.), Advances in child development and behavior, (Vol. 16). New York: Academic Press.

CABE, P. A. (1976). Transfer of discrimination from solid objects to pictures by pigeons: A test of theoretical models of pictorial perception. Perception \& Psychophysics, 19, 545-550.

Caelli, T. (1982). On discriminating visual textures and images. Perception \& Psychophysics, 31, 149-159.
Cerella, J. (1977). Absence of perspective processing in the pigeon. Pattern Recognition, 9, 65-68.

Corballis, M. C., Beale, I. L. (1976). The psychology of left and right. Hillsdale, NJ: Erlbaum.

D'Amato, M. R., Salmon, D. P., \& Colombo, M. (1985). Extent and limits of the matching concept in monkeys. (Cebus apella). Journal of Experimental Psychology: Animal Behavior Processes, 11, 35-41.

Delius, D. J., \& NowaK, B. (1982). Visual symmetry recognition by pigeons. Psychological Research, 44, 199-212.

Fox, J. (1975). The use of structural diagnostics in recognition. Journal of Experimental Psychology: Human Perception \& Performance, 1, 57-67.

HaRvey, L., \& Gervais, M. (1981). Visual texture perception and Fourier analysis. Perception \& Psychophysics, 24, 534-542.

HERRNSTEIN, R. J. (1979). Acquisition, generalization, and discrimination reversal of a natural concept. Journal of Experimental Psychology: Animal Behavior Processes, 5, 116-129.

Hoffman, J. E. (1978). Search through a sequentially presented visual display. Perception \& Psychophysics, 23, 1-11.

Julesz, B. (1981). Textons, the elements of texture perception, and their interactions. Nature, 290, 91-97.

Julesz, B., \& Bergen, J. R. (1983). Textons, the fundamental elements in preattentive vision and perception of textures. The Bell System Technical Journal, 62, 1619-1645.

Julesz, B., Gilbert, E. N., ShePP, L. A., \& Frisch, H. L. (1973). Inability of humans to discriminate between visual textures that agree in second-order statistics-revisited. Perception, 2, 391-405.

LEA, S. E. G., \& RYAN, C. M. E. (1983). Feature analysis of pigeons' acquisition of concept discrimination. In M. L. Commons, R. J. Herrnstein, \& A. R. Wagner (Eds.), Quantitative analyses of behavior: Discrimination processes. Cambridge, MA: Ballinger.

Lombardi, C. M., FaChinelli, C. C., \& Delius, J. D. (1984). Oddity of visual patterns conceptualized by pigeons. Animal Learning \& Behavior, 12, 2-6.

Martin, R. C., \& Pomerantz, J. R. (1978). Visual discrimination of texture. Perception \& Psychophysics, 24, 420-428.

Podgorny, P., \& GARner, W. R. (1979). Reaction time as a measure of inter- and intraobject visual similarity: Letters of the alphabet. Perception \& Psychophysics, 26, 37-52.

Premack, D. (1978). On the abstractness of human concepts: Why it would be difficult to talk to a pigeon. In S. H. Hulse, H. Fowler, \& W. K. Honig (Eds.), Cognitive processes in animal behavior. Hillsdale, NJ: Erlbaum.

Reicher, B., SNyder, C., \& Richards, J. (1976). Familiarity of back ground characters in visual scanning. Journal of Experimental Psychology: Human Perception \& Performance, 2, 522-530.

SAS InSTITUTE (1982). SAS system [Computer program]. Cary, NC: Author.

Shepard, R. N., \& Arabie, P. (1979). Additive clustering: Representation of similarities as combinations of discrete overlapping properties. Psychological Review, 80, 87-123.

Todrin, D. C., Blough, D. S. (1983). The discrimination of mirrorimage forms by pigeons. Perception \& Psychophysics, 34, 397-402.

Triesman, A. M., \& Gelade, G. (1980). A feature-integration theory of attention. Cognitive Psychology, 12, 97-136.

TVERSKY, A. (1977). Features of similarity. Psychology Review, 84, 327-352.

Zentall, T. R., Hogan, D. E., Edwards, C. A., \& Hearst, E. (1980). Oddity learning in the pigeon as a function of the number of incorrect alternatives. Journal of Experimental Psychology: Animal Behavior Processes, 6, 278-299.

(Manuscript received July 8, 1985; revision accepted for publication October 22,1985 .) 